\title{
CÂNCER DE PULMÃO
}

\author{
LUNG CANCER
}

César Uehara'; Sérgio Jamnik² \& Ilka Lopes Santoro²

${ }^{1}$ Docente da Disciplina de Pneumologia; ${ }^{2}$ Médicos Assistentes. Departamento de Medicina da Escola Paulista de Medicina da UNIFESP. CorRESPONDÊNCIA: Disciplina de Pneumologia EPM / UNIFESP Rua Botucatu, 740, Vila Clementino, São Paulo-SP - CEP:04023-062. Tel: (011) 5491830.

UEHARA C; JAMNIK S \& SANTORO IL. Câncer de pulmão. Medicina, Ribeirão Preto, 31: 266-276, abr.jun. 1998

RESUMO: Este artigo contém os fundamentos básicos para o diagnóstico, tratamento e conduta dos pacientes portadores do carcinoma broncogênico. Nele, ainda, descrevemos os conhecimentos mais recentes que são discutidos na literatura mundial. É voltado para graduandos, pós-graduandos de Pneumologia, pneumologistas e generalistas.

UNITERMOS: Carcinoma Broncogênico.

\section{INTRODUÇÃO}

O câncer é um crescimento celular anormal, incontrolado, que invade os tecidos vizinhos e à distância, e é conhecido há vários séculos. Entre os de maior incidência encontra-se o câncer de pulmão, que, nos Estados Unidos, ocorreu em cerca de cento e setenta e sete mil (177.000) indivíduos, no ano de 1996. Ele é o segundo em incidência, após o câncer de próstata, no sexo masculino. Já, no sexo feminino, é o terceiro, após o câncer colorretal e o da mama.

É o câncer que mais leva a óbito, com cento e cinqüenta e oito mil e setecentas (158.700) mortes nos Estados Unidos, em 1996, independente do sexo. Quando todos os casos são considerados, aproximadamente 13 a 15\% sobrevivem num período de cinco (5) anos. É o único câncer cuja incidência de morte tem aumentado progressivamente, a despeito da melhora e da maior agressividade terapêutica nos últimos anos.

No Brasil, nos faltam melhores dados estatísticos, principalmente nas regiões mais pobres. Segundo a incidência de distribuição de óbitos na cidade de São Paulo, a neoplasia, em geral, ocupa a segunda posição com 16,2\%, sendo ultrapassada, apenas, pelas doenças do aparelho circulatório (Tabela 1).
Uma característica própria deste câncer de incidência alta de mortalidade é a sua causa, que é o hábito de fumar, o qual entretanto, possibilita uma ação profilática concreta e com bons resultados, que é o combate ao tabagismo. No nosso meio, começamos a observar esta tendência, que esperamos se intensifique nos próximos anos, vencendo os interesses econômicos que insistem em manter, entre as pessoas, o hábito de fumar. Neste capítulo, tentaremos, de forma sucinta, prática e abrangente discutir os principais fatores relacionados a esta doença.

\begin{tabular}{lr} 
Tabela I - Distribuição dos óbitos, segundo as \\
principais causas de morte, no município de São \\
Paulo. Período, outubro a dezembro de $\mathbf{1 9 9 5}$ \\
\hline Doenças do aparelho circulatório & $30,7 \%$ \\
Neoplasmas & $16,2 \%$ \\
Causas externas & $14,6 \%$ \\
Doenças do aparelho respiratório & $11,6 \%$ \\
Doenças endócrinas e metabólicas & $8,1 \%$ \\
Doenças do aparelho digestivo & $5,1 \%$ \\
Perinatais & $3,5 \%$ \\
Outras & $10,3 \%$ \\
\hline
\end{tabular}




\section{EPIDEMIOLOGIA}

Câncer de pulmão é a principal causa de morte por câncer, tanto em homens quanto em mulheres, nos Estados Unidos, sendo responsável por $28 \%$ de todas as mortes por câncer, em cada ano.

O padrão de incidência, devido à baixa sobrevida, é paralelo à taxa de mortalidade; 16 a 17\% de incidência, em homens, e 12\%, em mulheres, são atribuídos ao câncer de pulmão, sendo que a tendência desta incidência, de 1973 a 1990, demonstrou aumento, para mulheres, de $110,5 \%$ e de 9,2\% para os homens.

Em $85 \%$ dos casos o fumo é a causa da neoplasia, e o risco é associado à duração do hábito de fumar, ao número e tipo de cigarro fumado por dia.

\subsection{Epidemiologia descritiva}

\subsubsection{Idade e Sexo}

Nos Estados Unidos, as taxas de incidência idade-ajustada, no homem, atingiu platô e começou declinar em 1980, enquanto que, na mulher, continuou aumentando na década de 90.

A incidência idade-específica, no homem, foi máxima - 576,7/100000 - na faixa etária de setenta e cinco (75) a setenta e nove (79) anos e, na mulher, similar padrão foi evidenciado com risco de 233,4/100000 nas idades de setenta (70) a setenta e quatro (74) anos. O platô e declínio, após a idade de oitenta (80) anos para os homens e setenta (70) anos para as mulheres, é explicado pela baixa prevalência de tabagismo entre os homens antes de 1910, e para as mulheres, antes de 1920 .

\subsubsection{Raça}

O risco de câncer, em homens negros, foi $50 \%$ maior em homens brancos nos últimos dez (10) a quinze (15) anos. Enquanto que o risco, para mulheres negras, é de 10 a $20 \%$ maior que para mulheres brancas. A razão homem/mulher, nas taxas de incidência, reflete elevados riscos no homem entre 2,5 a 3,5/1.

As diferenças raciais nas taxas de incidência, são devidas ao padrão do cigarro fumado, com maior percentagem de nicotina, alcatrão e cigarros mentolados, assim como ao tempo de tabagismo.

\subsection{3 "Status" Sócio-Econômico}

Vários estudos demonstram associação inversa entre mortalidade e status sócio-econômico.

\subsubsection{Tipo Histológico}

A exposição ao tabaco aumenta o risco para carcinoma espinocelular, adenocarcinoma, carcinoma de grandes células e carcinoma indiferenciado de pequenas células de pulmão, porém tende a ser menor para os casos de adenocarcinoma.

\subsection{Fatores de risco}

\subsubsection{Tabaco}

A relação causal entre o hábito de fumar e câncer de pulmão foi estabelecida por estudos epidemiológicos, realizados nas décadas de 50 e 60 . A fumaça do cigarro tem mais de quarenta (40) agentes carcinogênicos, e essa combinação depende das condições ambientais do local onde o indivíduo está fumando, do uso de filtros, aditivos e do tipo de papel do cigarro.

A prevalência do hábito de fumar, para homens maiores de dezoito (18) anos, diminuiu de aproximadamente 54\% em 1985, para 28\%, em 1991; enquanto que, para mulheres, a diminuição da prevalência foi menor, passando de $33 \%$, em 1953, para $24 \%$ em 1991, nos Estados Unidos.

O risco de câncer de pulmão na população de fumantes é dezessete (17) vezes maior nos homens e onze (11) vezes maior nas mulheres, quando comparados com não fumantes. O método eficaz para redução da incidência da neoplasia de pulmão é estimular as pessoas não fumantes a continuarem não fumando e aos fumantes a pararem de fumar. O risco de câncer de pulmão diminui em ex-fumantes, sendo que, cinco anos após cessar o tabagismo, o risco cai pela metade, e, após dez (10) anos, cai a 1,4. Sabemos que, mesmo quinze (15) anos após parar de fumar, o exfumante ainda tem um risco para câncer de pulmão de 1,4 e 4 vezes maior que o não fumante.

\subsubsection{Fumante passivo}

O risco de não fumantes, expostos a fumaça de cigarro, para câncer de pulmão é de 1,2 a 1,5 maior que o do não fumante não exposto ao fumo dos seus pais ou cônjuges. A exposição de não fumantes à fumaça do tabaco, em ambiente de trabalho, é difícil de ser determinada.

\subsubsection{Radônio}

O radônio é um gás inerte, cujos produtos de degradação liberam partículas radioativas alfa, que interagem com o epitélio brônquico, aumentando a incidência de câncer de pulmão, tendo um risco de 1,3 , em casos de exposição a concentração média, e de 1,8, em casos de concentração mais elevada de radônio, em residências nos Estados Unidos, incidências estas similares às dos trabalhadores em usinas. 


\subsubsection{Exposição Ocupacional}

Trabalhadores expostos ao asbesto têm risco quadruplicado para câncer de pulmão e mesotelioma, que é ainda maior, quando associado ao hábito tabágico. Outras substâncias químicas industriais, associadas ao câncer de pulmão, são: arsênico, berílio, cádmio, clorometila-éter, hidrocarbrometos, gás mostarda e níquel.

\subsubsection{Predisposição Genética}

O risco pessoal para câncer de pulmão é aumentado mais que cinco (5) vezes, se pelo menos um dos pais morreu de câncer de pulmão. Em um estudo de famílias de mulheres com câncer de pulmão, foi observado: pessoas que nunca fumaram, com história familiar positiva, tinham risco de 5,7; fumantes com história familiar negativa, risco de 15,1 , e fumantes com história familiar positiva, risco 30,0.

Tal susceptibilidade pode ser dependente da interação gene-enzima competitiva, que afeta a ativação de pró-oncogenes e níveis de formação de apresentação do DNA. Observa-se polimorfismo do DNA no citocromo P450A1, relacionado ao carcinoma espinocelular, e pessoas com esta susceptibilidade genotípica tiveram risco relativo de 7,3, após ajuste para sua história tabágica.

\section{PATOlogia}

A classificação histológica do câncer de pulmão mais aceita pela literatura mundial é a da Organização Mundial de Saúde (OMS). Os quatro tipos histológicos principais que abrangem $95 \%$ dos casos são: carcinoma espinocelular, adenocarcinoma, carcinoma indiferenciado de pequenas células e carcinoma indiferenciado de grandes células. O diagnóstico diferencial mais importante é distinguir o carcinoma indiferenciado de pequenas células dos outros três tipos, que são conhecidos como carcinoma de não pequenas células, pois diferem quanto, ao tipo de tratamento, de forma radical. Patologistas experientes podem fazer a distinção em 85 a $90 \%$ das vezes. Os tipos mais comuns de neoplasia de pulmão são o carcinoma espinocelular e o adenocarcinoma, representando 40 e $30 \%$, respectivamente, o carcinoma indiferenciado de pequenas células varia de 15 a $20 \%$, e o carcinoma indiferenciado de grandes células, aproximadamente $10 \%$. Outros tipos histológicos incluem tumores mistos, tumores carcinóides, mesoteliomas, sarcomas.

\section{QUADRO CLÍNICO}

Como mais de $85 \%$ dos pacientes com câncer de pulmão morrem nos primeiros cinco anos pós-diagnóstico, a importância do diagnóstico precoce, em estádio inicial, especialmente no estádio I, onde a sobrevivência é de 60 a $90 \%$ com o tratamento cirúrgico, deve ser realçada.

A presença de sintomas é sinal de mau prognóstico. Observa-se $25 \%$ de sobrevida em cinco (5) anos para pacientes com neoplasia de pulmão, sintomáticos, enquanto que foi de $56 \%$ para os assintomáticos.

\subsection{Assintomáticos}

Em teoria, um indivíduo com câncer de pulmão começa com uma única célula maligna e a divisão celular se segue em uma razão constante de duplicação. Assim, $1 \mathrm{~cm}$ do tumor representa trinta (30) duplicações e um (1) bilhão de células. A identificação, neste volume celular, é difícil, porque Rx de tórax, raramente, detecta tumores com menos de $1 \mathrm{~cm}$.

Fumantes com doença pulmonar obstrutiva crônica têm maior risco de câncer que aqueles com função pulmonar normal. Observa-se que a taxa de risco para neoplasia de pulmão, entre aqueles indivíduos com $\mathrm{VEF}_{1}$ menor que $60 \%$ do previsto, foi de 6,07, quando ajustada para a carga tabágica.

\subsection{Sintomáticos}

A presença de sintomas na época do diagnóstico varia de $40 \%$, em população de "screening" de massa, a $98 \%$. Os sintomas podem ser causados por extensão local, metástases e efeitos paraneoplásicos ou sistêmicos.

\subsubsection{Efeitos Locais}

\subsubsection{Tosse}

Tosse é o sintoma mais comum (45 a $75 \%$ ). A fístula traqueoesofágica deverá ser lembrada em pacientes com tosse paroxística violenta, após alimentação; esta complicação é mais comum nos casos de tumor de esôfago que nos casos de tumor de pulmão. Tosse com grandes volumes de secreção mucóide pode estar presente em carcinoma bronquioalveolar.

\subsubsection{Hemoptise}

A incidência de hemoptise está entre 27 a 57\%. A hemoptise maciça é pouco freqüente, mas foi observado, que em hemoptise maciça, $20 \%$ tem carci- 
noma broncogênico e, destes, 50\% morrem. Estes óbitos foram atribuídos à asfixia.

\subsubsection{Dor Torácica}

Dor torácica está presente em 27 a 49\% dos casos de neoplasia de pulmão. O tipo de dor é freqüentemente intermitente do lado do tumor, tornando-se intensa e persistente devido à extensão para mediastino, pleura ou parede torácica.

\subsubsection{Sibilos ou Estridor}

Sibilos localizados sugerem obstrução do brônquio, enquanto que obstrução da grande via aérea produz estridor. Neste último, a análise da curva fluxo-volume pode ser útil, na identificação de lesão de traquéia ou laringe.

\subsubsection{Dispnéia}

Dispnéia está presente em aproximadamente $37 \%$ da população com câncer de pulmão. As causas de dispnéia incluem obstrução de grande via aérea, intrínseca e extrínseca, pneumonite obstrutiva ou atelectasia, derrame pleural, linfangite, derrame pericárdico, embolia tumoral, tromboembolismo, e condições comórbidas, como doença pulmonar obstrutiva crônica e insuficiência cardíaca.

\subsubsection{Efeitos Metastáticos}

Metástases extrapulmonares para câncer de pulmão ocorrem mais frequientemente para o fígado, supra-renais, ossos e cérebro.

\subsubsection{Rouquidão e Paralisia de Diafragma}

Rouquidão, como primeiro sintoma, ocorre em $18 \%$ dos pacientes. Paralisia da corda vocal esquerda é a mais comum, devido à localização e trajeto do nervo laríngeo-recorrente esquerdo. A paralisia de corda vocal bilateral é mais rara.

Paralisia diafragmática é usualmente assintomática, é achado radiológico. A paralisia de diafragma, em 1/3 dos casos, teve o câncer de pulmão como causa.

\subsubsection{Derrame Pleural}

Sabe-se que em torno de $7 \%$ dos pacientes com câncer de pulmão estão envolvidos com derrame pleural maligno. Estes derrames pleurais são exudatos sero-sanguinolentos e são, comumente, associados com adenocarcinomas.

Nem todos os derrames pleurais, em pacientes com neoplasia de pulmão, são devidos a metástases pleurais, outras causas são: obstrução linfática, pneumonite pós-obstrutiva ou atelectasia e infarto pulmo- nar. Portanto se faz necessário realizar toda uma propedêutica para o diagnóstico de infiltração.

\subsubsection{Síndrome de Pancoast}

A síndrome de Pancoast, nos casos de tumor do sulco superior, caracterizada por dor, síndrome de Horner, destruição óssea, a atrofia de músculos da mão. O sulco superior é um sulco criado pela passagem da artéria subclávia até a pleura dos ápices dos lobos superiores.

Os carcinomas não pequenas células são as causas mais freqüentes de tumor do sulco superior.

\subsubsection{Síndrome da Veia Cava Superior}

Obstrução ao fluxo sangüíneo na veia cava superior. Os sintomas mais comuns são: sensação de aumento de volume da cabeça e dispnéia. A tosse, dor e dispnéia são menos freqüentes. Os principais sinais clínicos incluem dilatação de veias do pescoço, circulação colateral na parede torácica anterior, edema facial e, em extremidades superiores, "facies" pletórica. Carcinoma broncogênico é a causa de 65 a $80 \%$ dos casos desta síndrome e ela representa $4 \%$ das manifestações clínicas.

\subsubsection{Coração}

Em estudos "post-mortem", metástase no pericárdio e derrame no pericárdio ocorreram em $30 \%$, e todos os tipos histológicos podem evoluir com esta manifestação clínica. Os sintomas e sinais mais comuns são: dispnéia, tosse, pulso parodoxal, estase jugular e aumento da área cardíaca.

\subsubsection{Fígado}

Metástases hepáticas estão presentes, em casos de necrópsias, em cerca de $30 \%$ dos casos de carcinoma espinocelular e de $60 \%$ do carcinoma de pequenas células. No período do estadiamento, as metástases hepáticas são encontradas em $26 \%$ dos pacientes.

Inicialmente, os casos são assintomáticos. Aumento do volume do fígado, nódulos hepáticos palpáveis podem estar presentes. Icterícia e ascite são infreqüentes. Alterações bioquímicas (TGO e TGP) podem identificar casos assintomáticos de metástases hepáticas.

\subsubsection{Supra-renal}

Em autópsias, metástases supra-renais estão presentes em 25 a $40 \%$ de pacientes com câncer de pulmão, mas a insuficiência de supra-renal é raramente observada clinicamente. Menos da metade das massas de adrenal são malignas, portanto é necessário confirmar o diagnóstico com biópsia com agulha. 


\subsubsection{8 Óssea}

Metástase óssea é freqüente, em especial para carcinoma de pequenas células; os corpos vertebrais são os mais freqüentemente envolvidos, usualmente ela é osteolítica. Sabe-se que, em carcinoma não pequenas células, $34 \%$ de mapeamento ósseo é alterado, porém apenas $8 \%$ confirmaram ser lesão maligna pela biópsia óssea.Um dos indicadores clínicos de metástases é a dor torácica, a sensibilidade óssea, e a elevação dos níveis de fosfatase alcalina ou cálcio sérico.

\subsubsection{Sistema Nervoso Central}

Pacientes com metástases cerebrais podem ser assintomáticos ou ter sintomas não focais, como dor-de-cabeça, náusea, vômito, tontura ou, então, sintomas focais de hemiparesias, déficit de nervos cranianos ou perda de campo visual. Em autópsias, estas metástases estão freqüentes, em um percentual de 26 a $38 \%$. O tipo histológico mais propenso a desenvolver este tipo de metástase é o carcinoma de pequenas células e o que menos freqüentemente envolve o sistema nervoso central é o carcinoma espinocelular. Em 10\% dos carcinomas de pequenas células, a metástase cerebral já estava presente no momento do diagnóstico.

Também são freqüentes causas de compressão extradural da medula espinal. A meningite carcinomatosa pode ocorrer.

\subsubsection{Síndromes Paraneoplásicas}

Ocorrem em 10 a 20\% dos pacientes, sendo mais comuns naqueles portadores de carcinoma indiferenciado de pequenas células.

- Caquexia e anorexia: causadas por efeitos locais do câncer, efeitos sistêmicos que afetam apetite e metabolismo, assim como efeitos do tratamento.

- Manifestação músculo-esquelética: como osteoartropatia hipertrófica, poliomiosite-dermatomiosite, etc.

- Síndromes hematológicas: anemia ferropriva, alteração da coagulação, aumentando a incidência de tromboembolismo

- Secreção inapropriada de hormônio antidiurético.

- Hipercalcemia: que pode ser devida a metástase óssea ou secreção de parato hormônio "like" pelo tumor. Presença de hipercalcemia sem lesão óssea metastática é mais comumente encontrada em carcinoma espinocelular.

- Síndrome de Cushing.

- Síndrome miastênica de Lambert-Eaton.

\section{TÉCNICAS DIAGNÓSTICAS}

Pacientes com sinais e sintomas compatíveis com neoplasia de pulmão devem ser imediatamente investigados para se chegar ao diagnóstico definitivo o mais precocemente possível. É necessário entender o mérito específico e a limitação de cada procedimento diagnóstico (especificidade, sensibilidade, fatores de risco e custo econômico) para otimizar a escolha e seqüência dos exames.

\subsection{Radiografia de Tórax}

Ainda é a "chave" para detecção de câncer de pulmão. Tem alta sensibilidade para tumores periféricos, baixo custo e risco insignificante. Deve ser pedido sempre nas posições póstero-anterior e perfil.

As imagens radiológicas são variáveis, e carcinoma de pulmão deve ser considerado como diagnóstico diferencial para qualquer nódulo pulmonar não calcificado, em especial, em homens fumantes, lembrando que a presença de cálcio na lesão não é sinônimo de benignidade.

Para se confirmarem lesões em costelas, será necessária a técnica com baixa kilovoltagem (60 a 80) em posição oblíqua.

\subsection{Tomografia Computadorizada de Tórax}

Acrescenta muito para o diagnóstico e estadiamento em neoplasias de pulmão. Este exame nos dá noções precisas do tamanho, localização e níveis de invasão do tumor; também em relação aos diferentes níveis das cadeias ganglionares do mediastino.

No estudo tomográfico, abrangendo andar superior do abdome, analisar-se-ão o fígado e as supra-renais, que são sítios importantes, pela freqüência, de lesões metastáticas.

\subsection{Ressonância Magnética}

No momento atual, este exame acrescenta muito pouco à tomografia computadorizada de tórax, e é um equipamento caro.

A principal vantagem é distinguir estruturas vasculares de estruturas sólidas, sem o uso de contraste, dando maior precisão ao estudo do mediastino. Também identifica muito bem a extensão do tumor no forame mural, assim como invasão tumoral em parede torácica e pericárdio.

\subsection{Citologia de Escarro}

A coleta do material deve ser feita pela manhã, em dias consecutivos. É mais sensível para os tumo- 
res centrais. Um citopatologista experiente consegue determinar o tipo histológico em $80 \%$ dos casos positivos. A diferenciação entre carcinoma de pequenas células e do grupo carcinoma não pequenas células tem alto nível de confiabilidade.

\subsection{Broncofibroscopia}

É a técnica definitiva para o diagnóstico, pois permite a visualização da lesão, quer seja em traquéia, quer em brônquios, como também colher material em forma de lavado broncoalveolar, escovado brônquico e biópsias brônquicas e transbrônquicas. Em casos de lesões subsegmentares, a escova ou pinça de biópsia podem ser locadas com o auxílio de fluoroscopia, aumentando o rendimento do exame.

O broncofibroscópio é usado em $95 \%$ dos exames, enquanto o aparelho rígido é reservado para situações especiais.

A acurácia do exame é alta para os tumores centrais, variando de 55 a $80 \%$ para todos os tumores. As lesões metastáticas, em pulmão, são menos sensíveis ao diagnóstico broncoscópico.

A punção realizada por agulha de aspiração transbrônquica permite a obtenção de material de gânglio subcarinal e nódulos periféricos para análise citológica.

\subsection{Biópsia por Agulha Transcutânea}

A fluoroscopia e a tomografia computadorizada de tórax são os principais auxiliares para este procedimento. Tem grande acurária (74 a 96\%) em mãos experientes. Assim, a sensibilidade do exame depende do médico que o executa, do citologista e da seleção da lesão.

O risco de pneumotórax está entre 20 a 35\%, mas apenas 5 a $10 \%$ necessitam de drenagem torácica.

Como contra-indicação, temos pacientes com risco aumentado para pneumotórax (inabilidade para colaborar com o exame, tosse incontrolável, lesão central) e com risco aumentado de hemorragia (diátese hemorrágica e hipertensão pulmonar).

\subsection{Mediastinoscopio}

Método usado para melhorar o estadiamento ao mediastino, em casos de suspeita de lesão ganglionar em mediastino (N2).

\subsection{Biópsia a Céu Aberto}

Está indicada todas as vezes que os métodos anteriores, menos invasivos, falharam em dar o diagnóstico.

\subsection{Toracocentese}

Indicado em casos de derrame pleural, para realização de citologia do líquido, com sensibilidade entre 40 a $70 \%$, principalmente para adenocarcinoma (por ter mais esfoliação das células pleurais).

A sensibilidade do citológico será tanto melhor quanto maior for o número de técnicas utilizadas para a coloração das lâminas (HE, Leisheman) e o número de lâminas feitas.

Outra técnica a ser realizada é a morfometria pelo sistema analisador de imagem computadorizada, assim como quantificar as regiões organizadoras nucleolares (Ag. Nor).

\section{ESTADIAMENTO}

\subsection{Carcinoma de Não Pequenas Células}

Baseado no sistema TNM, descrito para neoplasia pulmonar por Clifton F. Mountain ${ }^{(1)}$. No ano de 1997, o mesmo autor fez algumas modificações na descrição anterior, baseado em dados de diferença na sobrevida dos estádios (Tabela II).

\section{T - Descrição do tumor primário, onde:}

Tx - carcinoma oculto, encontrado em células malignas no escarro ou lavado brônquico, sem visualização do tumor primário.

T0 - nenhuma evidência do tumor primário (em geral - apresentação extratorácica).

Tis - carcinoma "in situ".

T1 - tumor menor de $3 \mathrm{~cm}$ no seu maior diâmetro, sem envolvimento da pleura visceral. À broncoscopia, o tumor não deve invadir brônquio lobar.

$\mathrm{T} 2$ - tumor maior de $3 \mathrm{~cm}$, invadindo pleura visceral, ou presença de atelectasia ou pneumonia obstrutiva; à broncoscopia, o tumor deve distar pelo menos $2 \mathrm{~cm}$ da carina principal ou estar em brônquio lobar.

T3 - tumor de qualquer tamanho com extensão para parede do tórax (incluindo tumores de sulco superior), diafragma, pleura mediastinal ou pericárdio sem envolver coração, grandes vasos, traquéia, esôfago ou corpo vertebral; ou um tumor em brônquio principal dentro dos $2 \mathrm{~cm}$, mas não invadindo carina principal, ou com atelectasia de todo o pulmão.

T4 - tumor de qualquer tamanho com invasão de mediastino, coração, grandes vasos, traquéia, esôfago, corpo vertebral ou carina principal, ou presença de derrame pleural maligno, ou nódulo no mesmo lobo do tumor primário. 


\section{N - Envolvimento Nodal}

N0 - nenhuma evidência de metástase em linfonodo.

N1 - metástases em linfonodos peribronquiais e/ou região hilar ipsilateral.

N2 - metástases em linfonodos mediastinais, ipsilateral ou subcarinal.

N3 - metástases em linfonodos hilares ou mediastinais contralaterais, ou escalênico ou subclavicular, ípsi ou contralateral.

\section{M - Metástases}

M0 - nenhuma metástase conhecida

M1 - presença de nódulo tumoral metástico em outro lobo que não o do tumor primário, metástases à distância.

\begin{tabular}{|c|c|c|c|}
\hline \multicolumn{4}{|l|}{ Estádios } \\
\hline \multicolumn{3}{|l|}{ estádio } & Mo \\
\hline 0 & Tis & & \\
\hline la & T1 & No & Mo \\
\hline $\mathrm{lb}$ & T2 & No & Mo \\
\hline Ila & T1 & N1 & Mo \\
\hline \multirow{3}{*}{ Ilb } & 厂 T2 & N1 & Mo \\
\hline & L T3 & No & Mo \\
\hline & $\tau^{\top 3}$ & N1 & Mo \\
\hline \multirow{3}{*}{ Illa } & T1 & N2 & Mo \\
\hline & T2 & N2 & Mo \\
\hline & CT3 & N2 & Mo \\
\hline \multirow[t]{2}{*}{ IIIB } & qualquer $\mathrm{T}$ & N3 & Mo \\
\hline & T4 & qualquer $\mathrm{N}$ & Mo \\
\hline IV & qualquer $\mathrm{T}$ & qualquer $\mathrm{N}$ & M1 \\
\hline
\end{tabular}

A escolha pela diferenciação do estádio I, em A e B, deve-se à sobrevida no estádio IA ser significantemente melhor que a dos pacientes IB, o mesmo ocorrendo com o estádio II.

\subsection{Carcinoma de Pulmão de Pequenas Células}

O sistema TNM não é aplicado rotineiramente neste grupo. Carcinoma de pulmão de pequenas células são classificados pelo sistema:

- Doença localizada: quando o tumor é confinado a um hemitórax e os linfonodos comprometidos são os mediastinais, supraclaviculares ipsilaterais e hilares contralaterais. Tumor que apresenta derrame pleural ipsilateral, envolvimento de nervo laringoesquerdo, obstrução de veia cava superior.

- Doença extensa: doença avançada localmente e à distância, incluindo envolvimento de pericárdio e parênquima bilateral.

\section{PROGNÓSTICO}

A correlação entre incidência de câncer de pulmão e mortalidade resulta de uma média de taxa de sobrevida num período de cinco (5) anos.

A proporção dos pacientes que morrem da doença (taxa de mortalidade) é maior que $90 \%$. Em relação aos outros cânceres, têm havido relativamente poucas mudanças na sobrevida, a despeito de intensa investigação na detecção precoce e de novos métodos de terapia cirúrgica, radiação e novas drogas quimioterapêuticas.

Nos Estados Unidos, as taxas de sobrevida no período de cinco (5) anos para todos os casos de câncer de pulmão foram: $6 \%$ de 1950 a 1954 e 13,4\% no período de 1981 a 1987 (National Câncer Institute). Taxas, estas, menores que as de quaisquer outros cânceres.

A redução na mortalidade, no próximo século, dependerá, primeiramente, da efetividade dos esforços para reduzir a prevalência do tabagismo.

O estadiamento dos carcinomas não pequenas células (CEC, adenocarcinoma e CIGC) é fator fundamental no prognóstico. À medida em que progredimos no estadiamento, menor será a sobrevida em cinco (5) anos (Tabela III).

\begin{tabular}{|c|c|c|c|}
\hline I & 60 a $85 \%$ & la & $60,7 \%$ \\
\hline II & 40 a $60 \%$ & $\mathrm{lb}$ & $37,6 \%$ \\
\hline IIla & abaixo $30 \%$ & Ila & $33,8 \%$ \\
\hline Illb & & $\mathrm{Ilb}$ & $23,4 \%$ \\
\hline \multirow{3}{*}{ IV } & menos $5 \%$ & IIla & $12,5 \%$ \\
\hline & & Illb & $4,6 \%$ \\
\hline & & IV & $1,1 \%$ \\
\hline
\end{tabular}

A mesma coisa ocorre com os descritores $\mathrm{T}$ (Tamanho Tumor), N (Nódulo Linfático) e M (Metástase), quando a sobrevida diminui com a progressão da doença, o que levou a recente modificação (1997) do estadiamento do câncer de pulmão. 
Isto também é válido no estadiamento dos carcinomas pequenas células, quando a sobrevida dos portadores de doença limitada é superior à dos portadores de doença disseminada. À presença de marcadores tumorais (Cmyc, Kras, p185, Cerb B-2) os pacientes têm apresentado menor sobrevida que a daqueles pacientes onde eles foram negativos. À presença de tumores diplóides, os pacientes têm menor sobrevida em cinco (5) anos que os que têm tumores aneuplóides (80\% x 20\% nos carcinomas não pequenas células). As mulheres têm, consistentemente, maior taxa de sobrevida que os homens.

\section{TRATAMENTO}

O tratamento de escolha para carcinoma broncogênico é a ressecção cirúrgica, porque é a modalidade que oferece a maior chance de cura.

Infelizmente, na maioria dos casos, o diagnóstico é feito em uma fase tardia, onde o tumor já se espalhou além dos limites de ressecabilidade. Assim, as outras formas de tratamento tornam-se de grande importância para a diminuição da morbidade e prolongamento da sobrevida.

Optaremos pela cirurgia, se o tipo histológico for carcinoma não pequenas células, se o estadiamento clínico for I, II ou, em alguns casos, III e se as funções cardiopulmonares não contra-indicarem o tratamento cirúrgico.

A ressecção cirúrgica de câncer de pulmão tem se tornado um procedimento seguro, com baixas taxas de complicações e mortes pós-operatórias. Um estudo com mais de dois mil (2.000) pacientes mostrou uma taxa média de mortalidade, após trinta (30) dias, de $3,7 \%$. Variando de $1,4 \%$ para pequenas ressecções (menos que uma lobectomia) a $6,2 \%$ para pneumectomia. As principais causas de morte no pós-operatório foram: insuficiência respiratória por pneumonia, fístula broncopleural com empiema, problemas cardíacos e embolia pulmonar.

No intra-operatório, o cirurgião optará, dependendo do estádio real do tumor e da necessidade de preservar a função pulmonar pela ressecção em cunha, segmentectomia, lobectomia ou mesmo uma pneumectomia com extensa ressecção dos nódulos linfáticos, responsáveis pela drenagem do tumor. Logo, o cirurgião deve tentar remover o mínimo de pulmão possível, associado com a completa ressecção do câncer.

Nós acreditamos que todos os pacientes submetidos a uma ressecção completa deveriam realizar pelo menos quatro (4) ciclos de quimioterapia, para combater as possíveis micrometástases. No nosso serviço, utilizamos a associação ifosfamida e a farmorrubicina.

Consideramos contra-indicação cirúrgica: obstrução da veia cava superior, paralisia da corda vocal, invasão do esófago, tumores de Pancoast, com invasão da coluna vertebral, e presença de derrame pleural com citologia classe $\mathrm{V}$, ou biópsia pleural com tumor, ou seja, estádios IIIb e IV.

Aos pacientes com metástases à distância há contra-indicação cirúrgica, sendo esta considerada uma exceção, quando o paciente apresenta uma lesão pulmonar ressecável e uma metástase solitária no cérebro ou fígado.

Só se realizará cirurgia em CIPC em duas condições: para se fazer diagnóstico de nódulo ou como adjuvante à quimioterapia. $\mathrm{O}$ tratamento principal do CIPC é a quimioterapia e, no nosso serviço, utilizamos carboplatina e vepeside.

A radioterapia é outra modalidade terapêutica, e ela é utilizada nas doenças localizadas ou em doenças disseminadas, visando, na última, o controle de determinada sintomatologia, por exemplo, um paciente com câncer de pulmão com dor óssea por metástase, ou metástase cerebral com sinais localizatórios. Com este tratamento, podemos ter alguns efeitos colaterais mais comuns: esofagite, pneumonite aquitínica, fibrose pulmonar, derrame pleural e dermatite.

A moderna quimioterapia iniciou-se com a descoberta da mostarda nitrogenada. Os resultados dependem do tipo histológico. O carcinoma indiferenciado de pequenas células responde melhor que tumores de qualquer outro tipo histológico, no pulmão.

A resposta do tumor depende de uma série de fatores: "performance status", peso inicial do paciente, extensão da doença na apresentação, número de sítios metastáticos conhecidos, sítios específicos das metástases e histologia.

Poucas drogas dão alguns benefícios e algumas, como os corticosteróides, encurtam a sobrevida (Tabela IV).

Atualmente se usa combinações de drogas, pois, assim, obtemos melhores respostas e sobrevida. O ideal é que cada droga aja independentemente, tenha diferentes toxicidades e um sinergismo na destruição das células tumorais. Isto é difícil de se obter.

Na prática, para realizarmos o tratamento, dividimos os pacientes em dois (2) grupos: carcinomas indiferenciados de pequenas células (CIPC) e os de não pequenas células (CEC, adenocarcinoma e CIGC). 
Tabela IV - Resposta em carcinoma de pulmão não pequenas células, na quimioterapia com drogas únicas

\begin{tabular}{lc}
\hline Droga & Resposta \\
\hline Mostarda nitrogenada & $24-33 \%$ \\
Ifosfamida & $26 \%$ \\
Mitomicina & $18 \%$ \\
Adriamicina & $9 \%$ \\
Etoposide & $9 \%$ \\
Cisplatino & $21 \%$ \\
Carboplatina & $10 \%$ \\
\hline
\end{tabular}

Nos CIPC, utilizamos o seguinte esquema: carboplatina e vepeside. Realizamos de quatro (4) a seis (6) ciclos, cada ciclo com intervalos de três (3) a quatro (4) semanas. Já nos não pequenas células, utilizamos a ifosfamida e farmorrubicina em quatro (4) ciclos, com o intervalo anterior.

Este número de ciclos será realizado em casos de resposta completa, resposta parcial ou doença estável; em caso de doença progressiva, na vigência de quimioterapia, suspendemos a medicação.

Nos pacientes com condição clínica boa, realizamos seguidamente Qt e Rd, pois teremos melhor controle da doença e a literatura nos mostra aumento da sobrevida.

Existe uma outra indicação para o uso de Qt, que é a terapia neo-adjuvante, utilizada nos carcinomas localmente avançados. Neste caso, realiza-se a Qt antes da cirurgia, visando diminuir o estadiamento do tumor, reduzir a recorrência local e diminuir a incidência de metástases à distância.

O grande problema da quimioterapia é o grande número de efeitos colaterais que se seguem a sua aplicação. Os principais são: anemia, granulocitopenia, podendo facilitar infecções em vários sítios, incluindo septicemia, mucosite, náusea, vômitos e alopecia. A intensidade de cada efeito colateral depende do tipo de droga utilizada. Com o desenvolvimento de novas drogas antieméticas, estimuladores de colônia e outros, os efeitos colaterais passaram a ser mais suportáveis.

Vários pesquisadores utilizam imunoterapias, com levamisole, BCG, corynebacterium parvum, interferon e, no nosso serviço, a glucana. Acreditava-se que a estimulação do sistema imune inibiria o crescimento tumoral, infelizmente até o momento, os resultados são controversos.

\section{DERRAME PLEURAL MALIGNO}

Tem grande significado clínico, se for a primeira manifestação, fonte de diagnóstico da doença, e nos fornece importantes informações prognósticas. Freqüentemente, ocorre em estádio avançado da doença. Os principais carcinomas a evoluírem com derrame são os de pulmão, mama, ovário, estômago e os linfomas, representando mais de $80 \%$. Ocorre devido a distúrbio do fluxo do líquido no espaço pleural, depois da implantação de células tumorais na pleura, por extensão direta, metástase linfática ou hematogênica. Os dois principais processos responsáveis pela formação do derrame pleural são:

- aumento da permeabilidade capilar, devido a inflamação e/ou ruptura do endotélio capilar,

- piora da drenagem linfática dentro do espaço pleural secundário à obstrução pelo tumor.

As principais manifestações clínicas são a dispnéia, tosse, dor torácica e sensação de peso no tórax, relatada em mais de $50 \%$ dos pacientes, sendo que menos de $25 \%$ são assintomáticos. Ao exame físico, encontramos, em 90\% dos casos, macicez, diminuição do frêmito toracovocal, egofonia e sinal de Signorelli positivo.

A suspeita é confirmada com radiograma de tórax em PA e perfil, onde observamos a presença da linha de Trousseou, se houver mais de $100 \mathrm{ml}$ de líquido no espaço pleural. Em caso de dúvidas, ou volume menor, poderemos utilizar a radiografia de tórax, em decúbito lateral sobre o lado comprometido, com raios horizontais, ou o ultra-som de tórax.

A toracocentese é imperativa para determinar as características químicas e celulares do líquido pleural.

Na maioria dos casos, observamos que se trata de exsudato com proteína superior a 3,0g/100 ml e DHL > 200U e relação fluidosérica igual ou superior a 0,5 e 0,6 , respectivamente.

A pesquisa da citologia oncótica torna-se importante pois na grande maioria, é classe V (presença de células neoplásicas). Para casos negativos (após duas (2) tentativas) deve-se realizar biópsia pleural. A associação dos dois exames nos dá sensibilidade próxima a $60 \%$. Caso a dúvida persista, deve-se fazer pleuroscopia, cuja sensibilidade se aproxima de $100 \%$.

Dependendo da perspectiva de sobrevida (alta) e da velocidade de recidiva do derrame pleural, o tratamento de escolha é pleurodese, que consiste na 
instilação de substâncias esclerosantes, que visam obliterar o espaço pleural e, com isso, não deixar acumular líquido, evitando-se os sintomas. Este tratamento é paliativo, e está contra-indicado na presença de atelectasia e drenagem torácicas inadequadas.

As principais substâncias esclerosantes descritas são: tetraciclina, bleomicina e talco, (Tabela V) infundidas através do dreno torácico, após pequena quantidade de xilocaína.

\begin{tabular}{|c|c|c|}
\hline Agentes & Dose & $\begin{array}{l}\text { Eficácia } \\
\text { após } 1 \text { mês }\end{array}$ \\
\hline Tetraciclina & $35 \mathrm{mg} / \mathrm{kg}(2,0 \mathrm{~g})$ & $69 \%$ \\
\hline Bleomicina & $60 U$ & $84 \%$ \\
\hline Talco (solução) & & $96 \%$ \\
\hline C. Parvum & $7 \mathrm{mg}$ & - \\
\hline
\end{tabular}

Nos tipos histológicos, sensíveis à quimioterapia (CIPC e linfomas) o tratamento com as drogas podem ser suficiente, para conter a recidiva do derrame pleural.

\section{NÓDULO PULMONAR SOLITÁRIO}

Caracterizado por lesão única, em forma de esfera, no parênquima pulmonar, apresentando, como limite superior, $3 \mathrm{~cm}$ no maior diâmetro, pois, acima deste tamanho, é considerado massa, embora alguns autores sugiram o limite de $6 \mathrm{~cm}$. Em aproximadamente 40 a 50\%, tais lesões são malignas, e, em sua maioria, carcinoma broncogênico. Nos casos de lesão benigna, predomina o granuloma de origem infecciosa, e hamatoma é mais raro.

Como boa parte dos nódulos solitários são carcinomas broncogênicos, a sua retirada resultando em alta taxa de sobrevida em cinco (5) anos, a toracotomia exploratória é freqüentemente considerada e indicada. Alguns critérios clínicos podem ajudar a pensar em benignidade e retardar uma possível toracotomia: 1) calcificação no nódulo (distribuição benigna). 2) estabilidade do nódulo observado por pelo menos dois (2) anos, através de radiografia do tórax ou 3) idade inferior a trinta e cinco (35) anos, em indivíduo não fumante.
A grande parte dos nódulos é detectada pelo Rx convencional de tórax, que nos permite a avaliação do tamanho, de calcificação no interior do nódulo, e pode servir de guia para biópsia de aspiração por agulha. Estudos seriados de Rx de tórax, permite avaliar se a lesão é estável, e, em conjunto com informações clínicas, supor quadro de benignidade. A tomografia computadorizada do tórax (TC) em especial a de cortes finos, permite estudo mais fidedigno do tamanho, calcificação, número e densidade da lesão. A presença de margens imprecisas, lobuladas, espiculadas e com baixo coeficiente de atenuação é sugestiva de malignidade.

O principal objetivo da biópsia é estabelecer que a lesão é benigna, e evitar a toracotomia. Infelizmente, em lesões menores que $3 \mathrm{~cm}$, a sensibilidade é bastante reduzida, principalmente quando se utiliza a broncoscopia como método para coleta de material. Apesar de uma positividade maior na biópsia por aspiração com agulha transtorácica, guiada por TC ou fluoroscopia, os resultados falso-negativos chegam até $29 \%$, não afastando definitivamente a presença de malignidade.

O tempo de duplicação, que é o tempo requerido para o nódulo dobrar de tamanho, em caso de nódulo maligno, varia de trinta (30) a quatrocentos (400) dias. Já em nódulo benigno, pode ter um crescimento variável, < 20 dias ou mais lento > 450 dias. Cálculo do tempo de duplicação, através de radiografias seriadas, é criticado, pois esta estratégia pode resultar na diminuição da taxa de sobrevida em cinco (5) anos, uma vez que este é inversamente relatado com o tamanho da lesão que é retirado com a toracotomia. A taxa de sobrevida é superior a $80 \%$, num período de cinco (5) anos, quando o tamanho do nódulo é menor do que $3 \mathrm{~cm}$ de diâmetro e há ausência de metástases nos nódulos linfáticos.

Mais recentemente, tem se utilizado a videotoracoscopia para a ressecção de nódulo pulmonar, diminuindo o tempo de internação e complicações no pós-operatório, embora este método não seja consenso entre todos os cirurgiões.

Como observamos, a decisão da conduta frente a um nódulo solitário do pulmão é difícil e duvidosa. A grande maioria dos pneumologistas levam em conta a pCA (probabilidade de câncer). Se for alta, deve-se realizar a toracotomia imediata; no entanto, se for baixa, pode-se esperar e observar. Outro aspecto a ser analisado é a ansiedade do paciente. 
UEHARA C; JAMNIK S \& SANTORO IL. Lung Cancer. Medicina, Ribeirão Preto, 31: 266-276, apr./june 1998.

ABSTRACT: The present article reviews basic concepts about diagnosis and treatment of lung cancer patients. The authors also discuss current aspects of the international literature in the field. This review is intended to medical students, pulmonologists and family doctors.

UNITERMS: Carcinoma, Bronchogenic.

\section{REFERÊNCIA BIBLIOGRÁFICA}

1 - MOUNTAIN CF. Anew international staging system for lung cancer. Chest 111: 1718-1723, 1997.

\section{BIBLIOGRAFIA RECOMENDADA}

1 - CORNEY DN et al. Cancer of the lungs. In: FISHMAN AP. Pulmonary diseases and disorders, 2nd ed., McGrawHill Book, New York, p.1885-2033, 1988.

2 - CARR DI; HOLOYE PY \& HONG WK. Bronchogenic carcinoma. In: MURRAY JF \& NADEL JA. Textobook of respiratory medicine, 2 ed. W.B. Saunders, Philadelphia, p. $1489-1622,1994$.
3 - GUIMARÃES CA et al. Câncer de pulmão, tumores pleurais costais; partes moles e outros. In: BETHLEM N. Pneumologia, 4 ed. Atheneu, São Paulo, p. 508-570, 1995

4 - JETT JR \& TAZELAAR HD. Lung cancer in the elderly. In: MAHLEY DA. Pulmonary disease in the elderly patients. MARCEL DEKKER, New York, p. 239-278, 1993.

5 - PARS HI et al. Lung cancer principles and practure, Lippincott - Raven, New York, p. 1-933, 1996.

Recebido para publicação em 13/05/98

Aprovado para publicação em 10/06/98 